\title{
Infiltração de água no solo em um plantio de melancia no sudeste paraense
}

\author{
Water infilltration in the soil in a watermelon plantation in southeast paraense \\ Infiltración de água en el suelo de una plantación de sandía en el paraense sureste
}

Recebido: 03/05/2021 | Revisado: 09/05/2021 | Aceito: 12/05/2021 | Publicado: 28/05/2021

\author{
Vanessa Silva Oliveira \\ ORCID: https://orcid.org/0000-0002-5403-1932 \\ Universidade Federal do Pará, Brasil \\ E-mail: voliveiraambiental@gmail.com \\ Eduarda Costa Ferreira \\ ORCID: https://orcid.org/0000-0001-9094-1151 \\ Universidade do Estado do Pará, Brasil \\ E-mail: eduardacostaferreira5@gmail.com \\ Virginia Bezerra Rodrigues \\ ORCID: https://orcid.org/0000-0001-5315-6517 \\ Universidade do Estado do Pará, Brasil \\ E-mail: virginiarodrigues89@gmail.com \\ Kelvis Nunes da Silva \\ ORCID: https://orcid.org/0000-0003-1085-5494 \\ Universidade Federal do Pará, Brasil \\ E-mail: kelvisnsilva@gmail.com \\ Gleidson Marques Pereira \\ ORCID: https://orcid.org/0000-0003-0657-7356 \\ Universidade do Estado do Pará, Brasil \\ E-mail: eng.gleidson.uepa@gmail.com
}

\begin{abstract}
Resumo
Objetivou-se no presente estudo realizar uma análise comparativa do comportamento da infiltração de água no solo em um plantio de melancia (Citrullus lanatus (Thunb.) Matsum \& Nakai). A área de estudo localiza-se no Projeto de Assentamento Ipiranga, no sudeste paraense. $\mathrm{Na}$ análise de infiltração aplicou-se o delineamento experimental inteiramente casualizado, com a utilização de dois aneis cilindros (25 e $50 \mathrm{~cm}$ de diâmetros cada e $30 \mathrm{~cm}$ de altura). Os dados de velocidade básica e infiltração de agua no solo foram obtidos in situ e comparados à dois modelos matemáticos empíricos. Os resultados médios de infiltração real obtidos foram 14,1582 $\mathrm{cm} \mathrm{h}^{-1} ; 5,4623 \mathrm{~cm} \mathrm{~h}^{-1}$; $13,3038 \mathrm{~cm} \mathrm{~h}^{-1}$ e $5,2538 \mathrm{~cm} \mathrm{~h}^{-1}$, respectivamente. A aplicação dos modelos empíricos elaborados por Kostiakov e Kostiakov-Lewis não representaram valores próximos ao real, porém Kostiakov-Lewis demonstrou melhor desempenho, principalmente em relação à Velocidade de Infiltração Básica (VIB). Concluiu-se a necessidade de rever a aplicação dos modelos de Kostiakov e Kostiakov-Lewis à ensaios de infiltração de água no solo com duração de tempo muito longo, pois os mesmos apresentam desvios não satisfatórios quando comparados ao valor real e sugerese a aplicação de outros modelos matemáticos empíricos, no entanto o fenômeno observado comparado ao cultivo de melancia apresentou lâminas acumuladas aceitáveis dentro de uma média de produção adequada para a região sudeste paraense.
\end{abstract}

Palavras-chave: Irrigação; Manejo; Umidade.

\begin{abstract}
The objective of this study was to perform a comparative analysis of the behavior of soil water infiltration in a watermelon (Citrullus lanatus (Thunb.) Matsum \& Nakai) plantation. The study area is located in the Ipiranga Settlement Project in southeastern Pará. In the infiltration analysis the experimental design was completely randomized, with the use of two cylindrical rings $(25$ and $50 \mathrm{~cm}$ in diameter and $30 \mathrm{~cm}$ in height). The data of basic velocity and infiltration of water were obtained in situ and compared to two empirical mathematical models. The

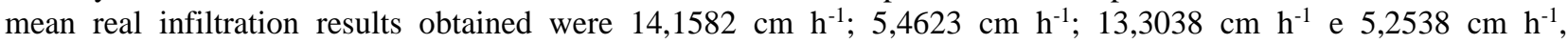
respectively. The application of the empirical models elaborated by Kostiakov and Kostiakov-Lewis did not represent values close to the real, but Kostiakov-Lewis demonstrated better performance, especially in relation to the Basic Infiltration Speed. It was concluded that there is a need to review the application of empirical models of Kostiakov and Kostiakov-Lewis to the tests of water infiltration in the soil with a very long time duration, since they have unsatisfactory deviations when compared to the real value and it is suggested the application of other empirical mathematical models, however the phenomenon observed compared to the watermelon crop showed acceptable accumulated slides within a production average suitable for the southeast region of Pará.
\end{abstract}

Keywords: Irrigation; Management; Moisture. 


\section{Resumen}

El objetivo de este estudio fue realizar un análisis comparativo del comportamiento de la infiltración de agua en el suelo en una plantación de sandía (Citrullus lanatus (Thunb.) Matsum \& Nakai). El área de estudio se ubica en el Proyecto Asentamiento Ipiranga, en el sureste de Pará. En el análisis de infiltración se aplicó un diseño experimental completamente al azar, con el uso de dos anillos cilíndricos ( 25 y $50 \mathrm{~cm}$ de diámetro cada uno y $30 \mathrm{~cm}$ de altura). Los datos básicos de velocidad e infiltración de agua se obtuvieron in situ y se compararon con dos modelos matemáticos

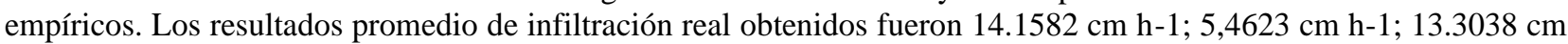
h-1 y $5.2538 \mathrm{~cm} \mathrm{~h}-1$, respectivamente. La aplicación de los modelos empíricos desarrollados por Kostiakov y Kostiakov-Lewis no representó valores cercanos al real, sin embargo Kostiakov-Lewis mostró un mejor desempeño, principalmente en relación a la Velocidad de Infiltración Básica (VIB). Se concluyó la necesidad de revisar la aplicación de los modelos de Kostiakov y Kostiakov-Lewis a ensayos de infiltración de agua en el suelo con una duración muy larga, ya que presentan desviaciones insatisfactorias al compararlo con el valor real y la aplicación se sugiere desde otros estudios En modelos matemáticos, sin embargo, el fenómeno observado en comparación con el cultivo de sandía mostró hojas acumuladas aceptables dentro de un promedio de producción adecuado para la región sureste de Pará.

Palabras clave: Riego; Gestión; Humedad.

\section{Introdução}

A melancia (Citrullus lanatus (Thunb.) Matsum \& nakai), pertence à família das cucurbitáceas, sendo originária do continente africano. É uma planta anual, de crescimento rasteiro, com várias ramificações que alcançam até $5 \mathrm{~m}$ de comprimento (Doorenbos \& Kassam, 1994). No Brasil, segundo dados IBGE (2017), a área plantada em 2017 superou 105.000 ha, com produção total de 2.314.700 toneladas de frutos e rendimento da ordem de 22 t/ha, sendo o estado do Pará responsável apenas por 3,82\% (88.495 t) dessa produção destacando-se (no Estado do Pará), a mesorregião sudeste (24.780 t), Pernambuco (122.360 t) e Maranhão (94.020 t).

A cultura da melancia adequa-se bem a solos de textura média, com boa drenagem e fertilidade favorável, mas as plantas também podem se desenvolver bem em solos com baixa capacidade de retenção de água e baixa fertilidade, para isso, as irrigações e fertigações devem ser realizadas com o objetivo de suprir as necessidades hídricas e de nutriente da planta (EMBRAPA, 2014).

As interações do solo com a água influenciam diversas funções ecológicas e ações de manejo do solo, pela determinação de quanto à água da chuva infiltra através do solo ou escorre sobre sua superfície. Dentre as funções ecológicas, destacam-se: a taxa de água perdida decorrente da lixiviação e evapotranspiração, o balanço entre ar e água nos poros, a taxa de variação na temperatura, a taxa e o tipo de metabolismo dos organismos e a capacidade em armazenar e disponibilizar água para as plantas (Molina Junior, 2017).

O manejo efetivo de sistemas de irrigação depende em grande parte do conhecimento de características físico-hídricas do solo, a infiltração de água no solo é um dos principais indicadores requeridos para dimensionamento, operação e avaliação de sistemas de irrigação. É definida como o processo pelo qual a água penetra no solo através da superfície (Pereira, 2007). O objetivo do presente estudo é comparar o comportamento da infiltração real com modelos matemáticos empíricos de água no solo em um plantio de melancia.

\section{Metodologia}

A metodologia utilizada trata-se de uma pesquisa dedutiva; de natureza aplicada; descritiva quanto aos objetivos; a abordagem do problema foi realizada de maneira quantitativa e quanto aos procedimentos técnicos é caracterizada como pesquisa de campo (Prodanov \& Freitas, 2013). 


\section{1 Área de estudo}

O estudo foi realizado no Lote 33 do Projeto de Assentamento Ipiranga (Figura 1), no Município de Curionópolis

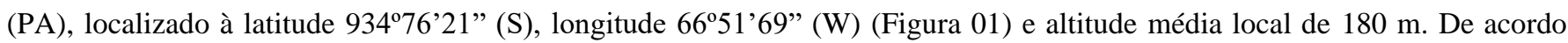
com a classificação de Koppen, a região apresenta clima do tipo quente e úmido com temperatura média anual de $26,3{ }^{\circ} \mathrm{C}$, e a precipitação média anual de aproximadamente $2.000 \mathrm{~mm}$ (IBGE, 2014). O solo do local foi classificado como Argilossolo Vermelho-Amarelo Distrófico com textura média/argilosa, de acordo com a metodologia da Embrapa (2018).

Figura 1 - Mapa de Localização da Área de Estudo.
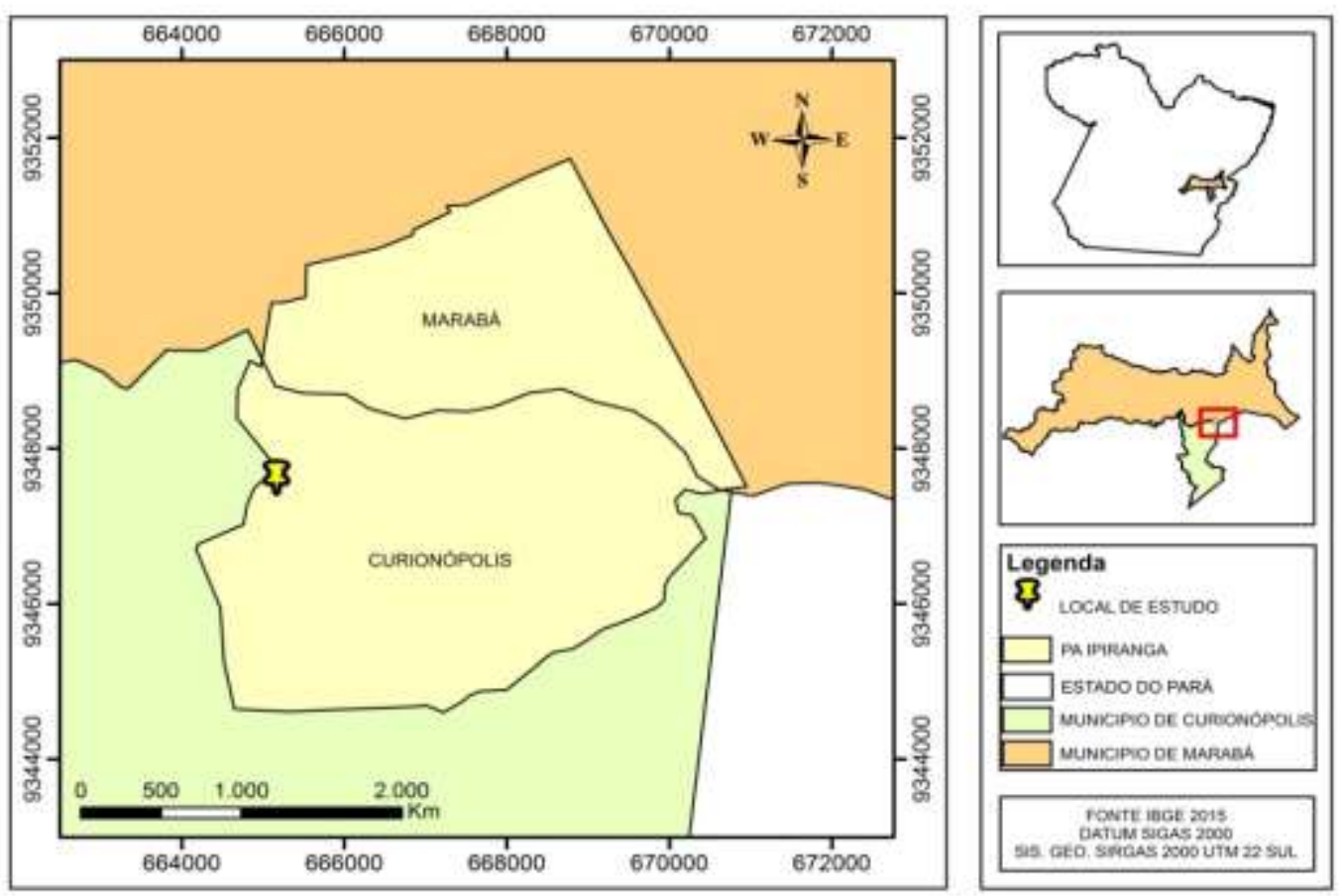

Fonte: Autores (2019).

\subsection{Amostragem e análise de infiltração}

Para análise de infiltração, utilizou-se o delineamento experimental inteiramente casualizado, foram realizados quatro ensaios em dias alternados com a duração de 2,5h. Utilizou-se a recomendação de Bernardo (1995): dois cilindros, um com 25 e outro com $50 \mathrm{~cm}$ de diâmetros, ambos com $30 \mathrm{~cm}$ de altura, instalados na vertical e enterrados a $15 \mathrm{~cm}$ de profundidade no solo. A leitura e determinação da água infiltrada são observadas no anel interno e a graduação do reservatório é compreendida em mm, enquanto o anel externo minimiza a dispersão de água no sentido lateral. A lâmina de água foi mantida numa média de 4 à $5 \mathrm{~cm}$ nos anéis, o teste teve a duração de 150 minutos.

Para o cálculo da VIB, foram utilizados o modelo empírico desenvolvido por Kostiakov (1932), de acordo com a metodologia proposta por Bernardo, Soares e Mantovani (2006), além do modelo de Kostiakov-Lewis (1945), ambos para descrever o volume de água que penetra no solo em função do tempo.

Modelo de Kostiakov (1942):

$$
\begin{aligned}
& I=a \times T^{n} \\
& \frac{d I}{d T}=V I=a \times n \times T^{n-1}
\end{aligned}
$$

Sendo que: 
I corresponde à infiltração acumulada $(\mathrm{cm})$

a e n são constantes que dependem do solo (AD)

VI equivale à velocidade de infiltração $\left(\mathrm{cm} \mathrm{h}^{-1}\right)$

T está relacionado ao tempo $(\mathrm{h})$

Modelo de Kostiakov Lewis (1945):

$$
\begin{aligned}
& I=a \times T^{n} \times k \times T \\
& \frac{d I}{d T}=V I=a \times n \times T^{n-1}+k
\end{aligned}
$$

Onde:

I corresponde à infiltração acumulada $(\mathrm{cm})$

$\mathrm{a}$ e $\mathrm{n}$ equivale às constantes que dependem do solo (AD)

VI é a velocidade de infiltração $\left(\mathrm{cm} \mathrm{h}^{-1}\right)$

Té o tempo (h)

K corresponde à velocidade de infiltração básica

As equações 1 e 3, compreendem as expressões para infiltração e 2 e 4, as expressões para Velocidade Básica de Infiltração (VBI).

\section{Resultados e Discussão}

\subsection{Infiltração}

Em relação à infiltração acumulada, verificou-se que o primeiro ensaio realizado, obteve uma taxa de infiltração bem superior aos demais, enquanto o terceiro e quarto ensaio, apresentaram valores mais próximos (Figura 2). As médias de infiltração, dos quatro ensaios foram: $14,1582 \mathrm{~cm} \mathrm{~h}^{-1} ; 5,4623 \mathrm{~cm} \mathrm{~h}^{-1} ; 13,3038 \mathrm{~cm} \mathrm{~h}^{-1}$ e 5,2538 $\mathrm{cm} \mathrm{h}^{-1}$, respectivamente.

Figura 2 - Infiltração Acumulada Observada In Situ.

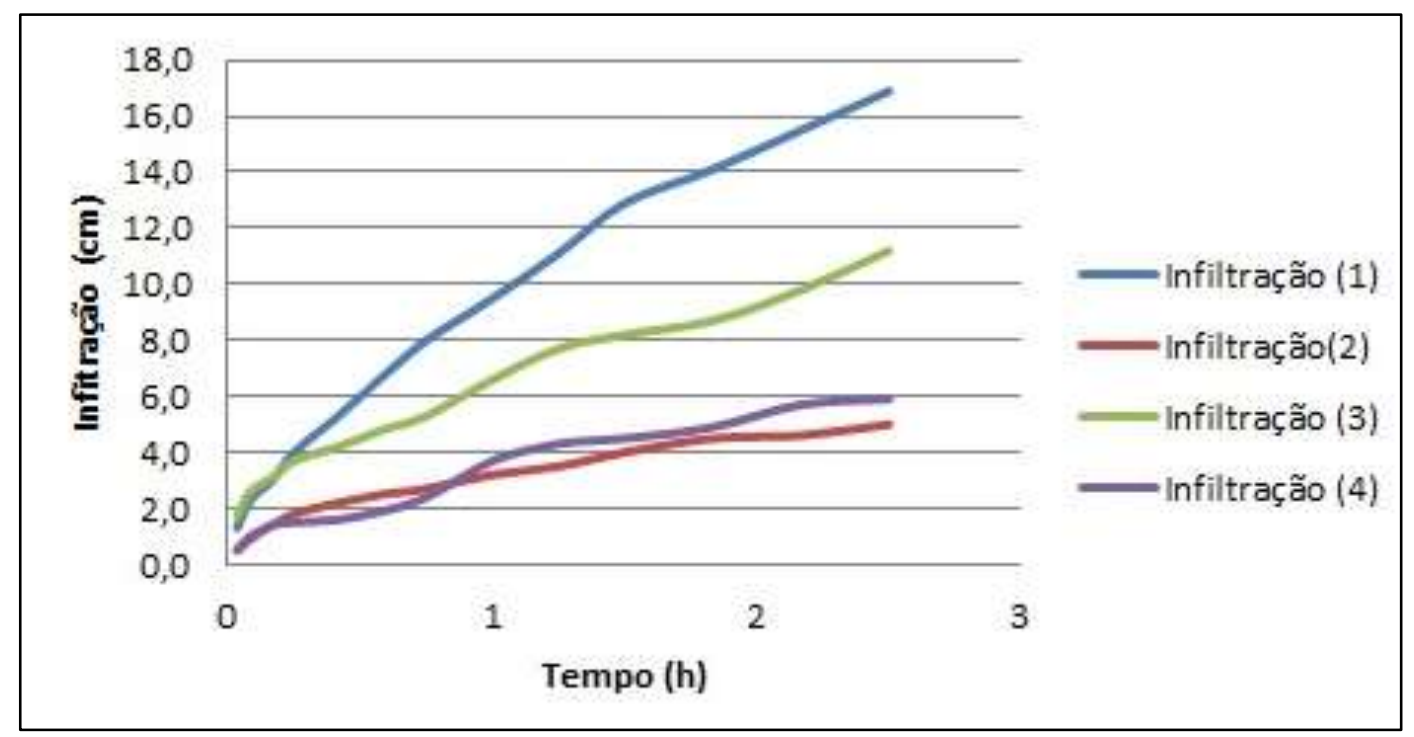

Fonte: Autores (2019). 
Reichardt e Timm (2004), afirmam que quanto mais seco o solo no início do processo de infiltração, mais alta será a taxa de infiltração, isto ocorre devido o gradiente de potência total ser enorme, o que pode explicar o fato das diferenças entre os ensaios. Nem sempre a condutividade do solo seco é maior do que a do solo úmido.

Através da visualização das linhas do gráfico, pode-se visualizar a equação exponencial da infiltração a partir da linha de tendência que derivando-a, obtêm se a velocidade. $\mathrm{O}$ indicador que o modelo se ajustou ao gráfico é representado por $\mathrm{R}^{2}$, o seu valor varia entre 0 e 1, quanto mais próximo de 1, mas bem ajustado ele se encontra. Os valores observados na imagem anterior equivalem a 0,$9968 ; 0,99 ; 0,9817$ e 0,981 , respectivamente.

Em relação à aplicação dos modelos empíricos elaborados por Kostiakov e Kostiakov-Lewis, nenhum representou valores próximos ao real, a princípio, a hipótese seria que o modelo de Kostiakov-Lewis poderia corrigir os valores calculados por Kostiakov, porém notou-se uma melhor representação (Figura 3).

Figura 3 - Comparação Média Entre a Infiltração Real e os Modelos Empíricos de Kostiakov e Kostiakov-Lewis (cm).

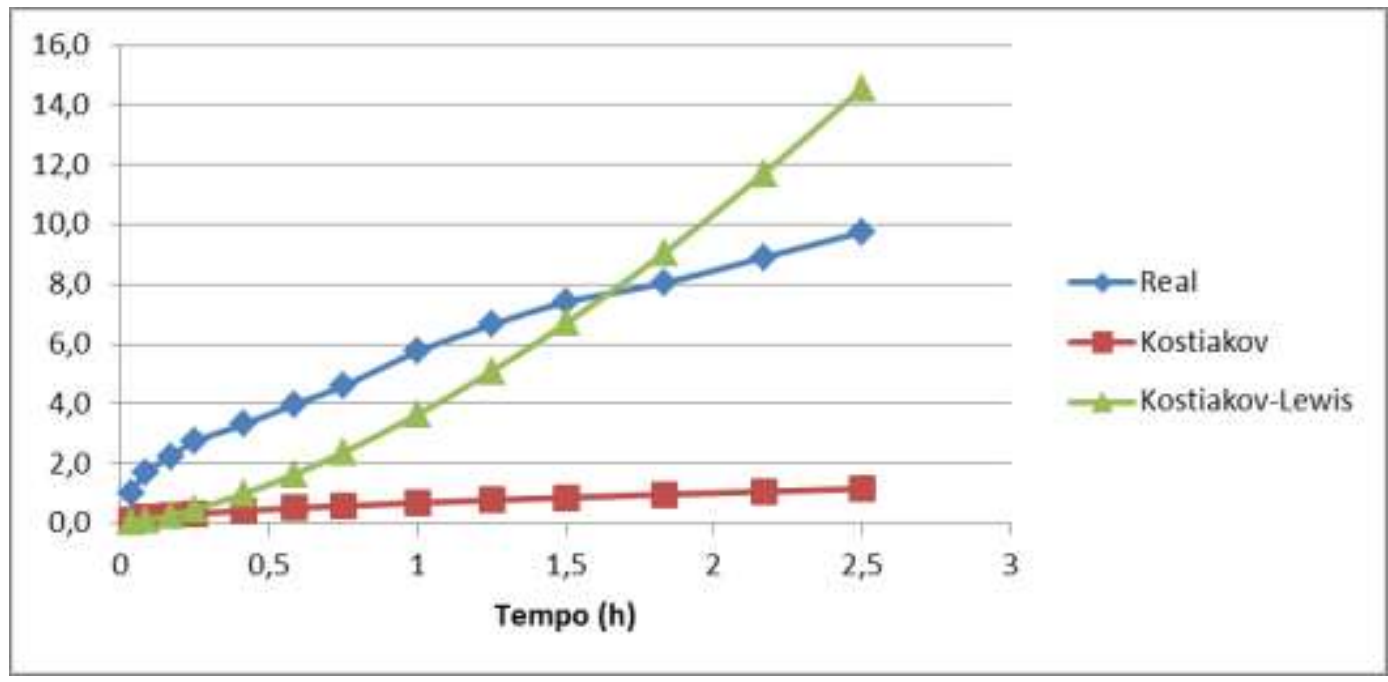

Fonte: Autores (2019).

Quando observado os valores médios, verificou-se entre os tempos de 1,5h e $2 \mathrm{~h}$ uma intercepção entre o resultado real e Kostiakov-Lewis.

Segundo Rocha (2010) a taxa de infiltração juntamente com a resistência do solo a penetração afeta diretamente o sistema radicular. Com isso as raízes tendem a espalhar na horizontal no caso da cultura de melancia, em solos com resistência a penetração baixa e alta taxa de infiltração. No caso desse experimento observa-se que a planta teria um bom desenvolvimento radicular indicando uma boa absorção de nutrientes pelas raízes.

\subsection{Velocidade de infiltração}

A Velocidade de Infiltração Básica - VIB de água, calculada com os dados de infiltração obtidos in situ são apresentados na Figura 4. 
Figura 4 - Velocidade Básica de Infiltração $\left(\mathrm{cm} \mathrm{h}^{-1}\right)$.

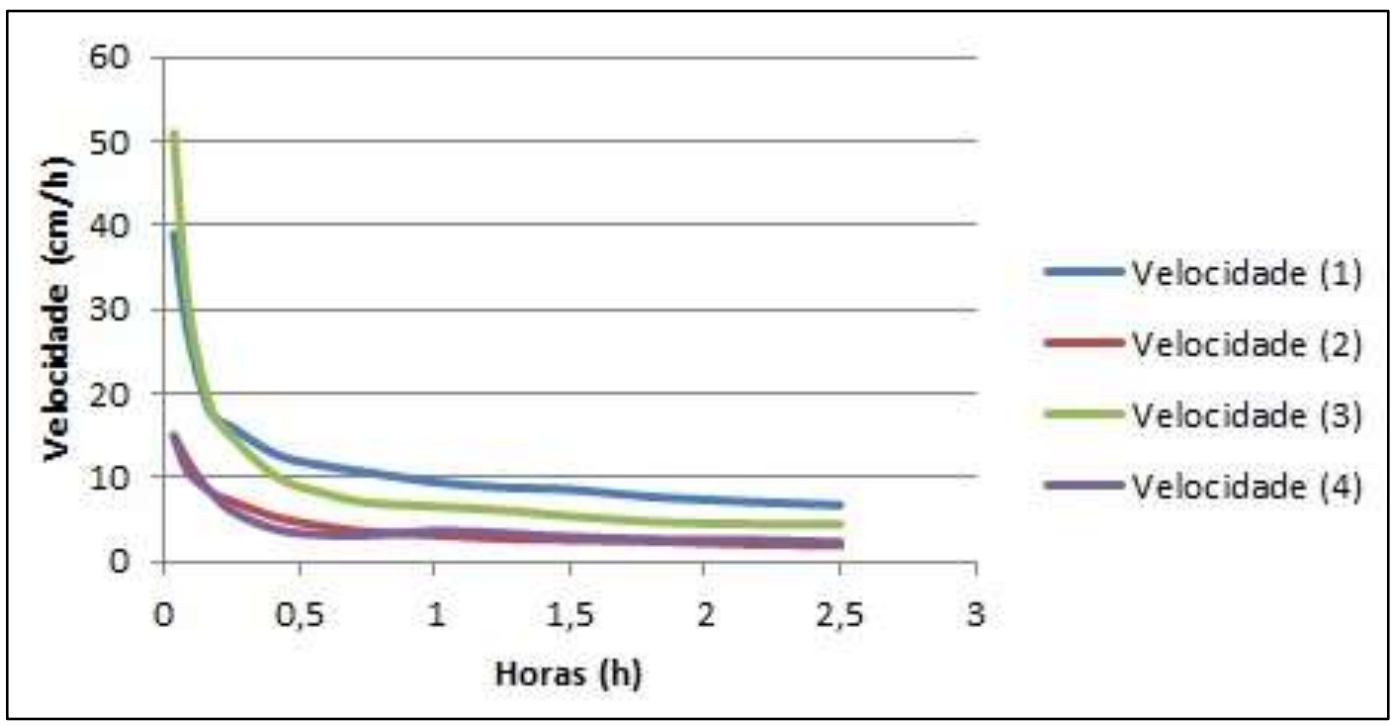

Fonte: Autores (2019).

As velocidades do primeiro e terceiro ensaio, e do segundo e quarto também tem resultados bem próximos, assim como pode-se observar anteriormente em relação à infiltração.

Da mesma maneira da aplicação dos modelos empíricos à infiltração, nenhum se comportou de maneira a representar a VIB real, porém Kostiakov-Lewis teve mais proximidade a partir de 1 hora de infiltração até o final das 2,5h, o que indica que uma quantidade de tempo muito grande de ensaio influencia na representação dos modelos quanto ao real (Figura 5).

Figura 5 - Comparação Média Entre a Velocidade Real $\left(\mathrm{Cm} \mathrm{H}^{1}\right)$ e os Modelos Empíricos de Kostiakov e Kostiakov-Lewis.

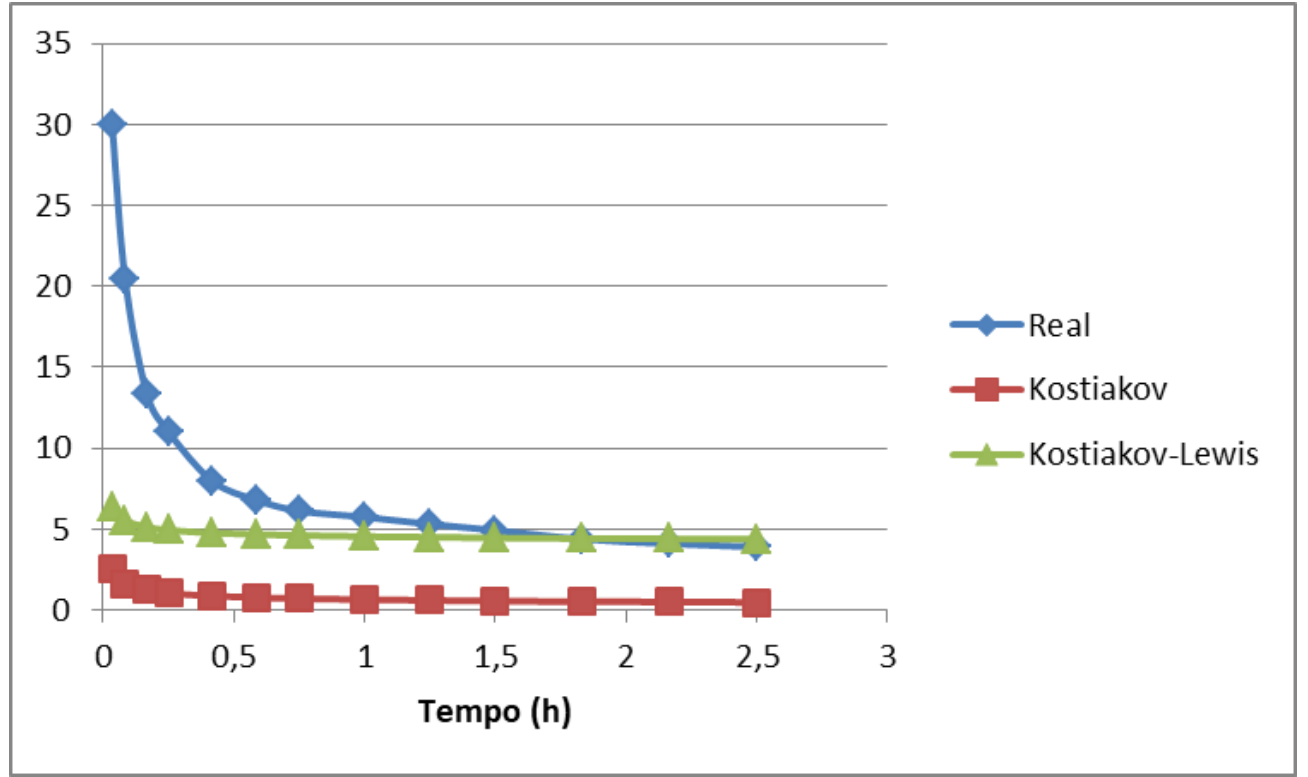

Fonte: Autores (2019).

Segundo Philip (1957) citado por Gomes Filho et al. (2018), a aplicação desses modelos empíricos é limitada para um tempo muito longo de infiltração, pois os dois parâmetros a e n são determinados a partir de leitura simultaneamente, porém, Kostiakov-Lewis representa melhor se comparada a Kostiakov. 
Os dados obtidos nesse estudo discordam de Paixão et al. (2009) que observou valores de dispersão bem menores em relação aos dois modelos empíricos, o autor destaca que a provável justicativa dessa dispersão é decorrente da grande heterogeneidade das propriedades físico-hídricas do solo, tais como: textura, densidade e umidade do solo, condutividade hidráulica, etc.

Bernardo (2002), afirma que solos quando apresentam valores de Velociade Básica (Vb) maiores que $3 \mathrm{~cm} \mathrm{~h}^{-1}$ são solos de $\mathrm{Vb}$ muito alta; valores entre 1,5 e $3 \mathrm{~cm} \mathrm{~h}^{-1}$, solos de alta $\mathrm{Vb}$; valores entre 0,5 e 1,5 $\mathrm{cm} \mathrm{h}^{-1}$, média $\mathrm{Vb}$; valores abaixo de $0,024 \mathrm{~cm} \mathrm{~h}^{-1}$, baixa Vb. Pelos valores reais e os calculados por Kostiakov-Lewis, considera-se a área estudada com solo de $\mathrm{Vb}$ muita alta.

A alta velocidade de infiltração de agua no solo quando somada a outros fatores ajuda a evitar formação de poços de água no terreno, já que o mesmo indica excesso de água causador do amarelamento da planta e a morte da mesma (SILVA, 2013). Na cultura da melancia é importante o controle do excesso de umidade já que o ambiente úmido é propicio a proliferação de fungos e consequentemente a ataques de pragas. Além disso, as rachaduras de frutos também estão relacionadas com a umidade principalmente a fase de maturação e temperatura elevada (acima de $35^{\circ} \mathrm{C}$ ) fazendo com que a preocupação com os limites de irrigação e o período de plantio seja de estrema importância para a qualidade do fruto e continuidade da cultura (EMBRAPA, 2007).

\section{Conclusão}

Necessita-se rever a aplicação dos modelos de Kostiakov e Kostiakov-Lewis à ensaios de infiltração de água no solo com duração de tempo muito longo, pois os mesmos apresentam desvios não satisfatórios quando comparados ao valor real e sugere-se a aplicação de outros modelos matemáticos empíricos, no entanto o fenômeno observado comparado ao cultivo de melancia apresentou lâminas acumuladas aceitáveis dentro de uma média de produção adequada para a região sudeste paraense.

Nessa conjuntura, é imprescindível que o trabalho não pode ser utilizado como referência única para outros trabalhos relacionados à temática de infiltração de água no solo, uma vez que cada cultivo se torna peculiar e pode influenciar no comportamento dessa infiltração, dado que cada solo pode se distinto além do tipo de cultivo no modelo de estudo, portanto, faz-se necessário que haja estudos mais precisos e elaborados, além de análises de outros parâmetros para o aprofundamento da pesquisa.

\section{Referências}

Bernardo, A. R. (1995). Manual de Irrigação. (6a ed.), Imprensa Universitária, 657.

Bernardo, S. (2002). Manual de Irrigação. (6a ed.), UFV.

Bernardo, S., Soares, A. A., \& Mantovani, E. C. (2006). Manual de Irrigação. (8a ed.), UFV, 625.

Doorenbos, J., \& Kassam, A. H. (1994). Efeito da água no rendimento das culturas. Estudos FAO: Irrigação e Drenagem. Universidade Federal da Paraíba: 306.

Empresa Brasileira de Pesquisa Agropecuária - EMBRAPA (2007). Melancia. Embrapa Meio-Norte. (2a ed.), Embrapa Informação Tecnológica, 85.

Empresa Brasileira de Pesquisa Agropecuária - EMBRAPA. (2014). Cultura da melancia. rev. Editora técnica Mirtes Freitas Lima. Embrapa Informação tecnológica.

Empresa Brasileira de Pesquisa Agropecuária - EMBRAPA. (2018). Sistema Brasileiro de Classificação do Solo. Embrapa Solos. (5a ed.), Embrapa.

Gomes F., et al. (2018). Análise do comportamento de modelos para ajuste da velocidade de infiltração em diferentes usos de solo. Revista Brasileira de Agricultura Irrigada. 12(4), 2755-60.

Instituto Brasileiro de Geografia e Estatística - IBGE (2019). Sistema IBGE de Recuperação Automática (SIDRA). https://sidra.ibge.gov.br/tabela/5457. 
Research, Society and Development, v. 10, n. 6, e21910615732, 2021

(CC BY 4.0) | ISSN 2525-3409 | DOI: http://dx.doi.org/10.33448/rsd-v10i6.15732

Instituto Brasileiro de Geografia e Estatística - IBGE (2014). Ministério do Meio Ambiente. Manuais técnicos em geociências: Ed.

Kostiakov, A. N. (1942). The Dynamics of the Coefficient of Water Percolation in Soils and the Necessity for Studying It from a Dynamic Point of View for Purpose of Amelioration. Society of Soil Science. 14, 17-21, https://www.scirp.org/(S(351jmbntvnsjt1aadkposzje))/refe rence/ReferencesPapers.aspx?ReferenceID $=1246023$.

Molina Junior, W. F. (2017). Comportamento mecânico do solo em operações agrícolas. ESALQ/USP, 223.

Paixão, F. J. R., Andrade, A. R. S., Azevedo, C. A. V., Costa, T. L., \& Guerra, H. O. C. (2009). Ajuste da curva de infiltração por meio de diferentes modelos empíricos. Pesquisa Aplicada \& Agrotecnologia, 2(1), 107-112.

Pereira, G. M. (2007). Análise comparativa de técnicas para estimativa da infiltração de água no solo em irrigação por superfície. 62f. Dissertação (Mestrado em Agronomia/Solos e Nutrição de Plantas) - Centro de Ciências Agrária, Universidade Federal do Ceará. Fortaleza.

Philip, J. R. (1957). Evaporation and moisture and heat fields in the soil. Journal of Meteorology. 14, 354-66, https://doi.org/10.1175/15200469(1957)014<0354:EAMAHF>2.0.CO;2.

Prodanov, C., \& Freitas, E. C. D. (2013). Metodologia do trabalho científico: métodos e técnicas da pesquisa e do trabalho acadêmico. (2a ed.), Editora Feevale.

Reichardt, K., \& Timm, L.C. (2004). Solo, planta e atmosfera: conceitos, processos e aplicações, 478. Barueri: Manole.

Rocha, M. R. (2010). Sistema de cultivo para a cultura da melancia, p.76. Dissertação (Mestrado em Ciência do Solo) - Centro de Ciências Rurais, Universidade Federal de Santa Maria.

Silva, M. G. O., et al. (2013). Manejo de plantas daninhas na cultura da melancia nos sistemas de plantio direto e convencional. Horticultura Brasileira, 31(3), 494-499. Vitoria da Conquista.

Taylor, H. M., \& Klepper, B. (1974). Water relations of cotton: Root growth and water use as related to top growth and soil water contetent. Agronomy Journal. 66(4), 584-8, Madison. 10.2134/agronj1974.00021962006600040031x. 